\title{
Endovascular Management of a Congenital Hepatic Arterioportal Malformation in a Low Resource Setting.
}

Poonamjeet Loyal ( $\sim$ kaurpoonamjeet@gmail.com )

Aga Khan University - Kenya https://orcid.org/0000-0001-8050-744X

\section{Ravjit Sagoo}

Aga Khan University - Kenya

\section{Research Article}

Keywords: congenital hepatic arterioportal fistula, metallic coil embolization.

Posted Date: February 8th, 2022

DOI: https://doi.org/10.21203/rs.3.rs-1199334/v1

License: (c) (i) This work is licensed under a Creative Commons Attribution 4.0 International License.

Read Full License 


\section{Abstract}

Background: Arterioportal malformations, a rare type of vascular malformation, have significant associated morbidity and mortality. Management requires a carefully thought out approach by a multidisciplinary team. Low resource settings have an added challenge of limited treatment options and consumables.

Case presentation: We report a case of a 14-month-old male with failure to thrive due to a congenital hepatic arterioportal fistula. He was successfully treated via an endovascular approach with metallic coil embolization.

Conclusion: Hepatoportal fistula, a rare hepatic vascular malformation, has limited treatment options which can further be restricted by overall patient wellness. Minimally invasive endovascular treatment options can offer a high rate of success and reverse the morbidity associated with the disease as was seen with our case.

\section{Introduction}

Hepatic arterioportal malformations is a communication seen directly between the portal vein and hepatic artery and can be divided into congenital and acquired. Congenital ones are a rare type of vascular malformation with one study reporting prevalence of less than $10 \%$ [1]. They may be associated with certain conditions including hereditary haemorrhagic telangiectasia, Ehlers-Danlos syndrome, and biliary atresia [2]. Acquired causes of hepatic arterioportal malformations include cirrhosis, neoplasms, trauma, and iatrogenic causes including liver biopsy, biliary surgery and gastrectomy $[3,4,5]$.

They are often asymptomatic but in rare instances can have debilitating symptoms that threaten survival. Some of the symptoms include portal hypertension, hepatofugal flow in the portal vein which becomes arterialized, splenomegaly, hypersplenism, variceal formation, bleeding, ascites, intestinal dysfunction including malabsorption, diarrhoea and esteatorrhea [5]. These patients are often extremely unwell and not ideal open surgical candidates. Therefore, minimally invasive treatment presents a suitable alternative.

\section{Case Report}

We present a case of a 14-month-old male who was referred to our facility with complete failure to thrive since birth and had developed melena stools intermittently for 1 month. The haemoglobin at presentation was $7.0 \mathrm{~g} / \mathrm{dl}$. The patient was seen at a peripheral facility but the referral to a specialist was delayed. An ultrasound and contrast-enhanced CT of the abdomen was performed which showed a $2.4 \mathrm{~cm} \times 2.2 \mathrm{~cm}$ tubular structure in the right hepatic lobe with early arterial filling from the left hepatic artery and draining into left portal vein (Figure 1 and 2). Early high flow retrograde arterial contrast filling of the left portal vein, the portal vein and the superior mesenteric vein, splenic vein and their tributaries was also seen. The 
portal vein, left portal vein, common hepatic and left hepatic artery were prominent. No other liver lesion was seen. There were also features of portal hypertension with periportal collateral vessels.

A multidisciplinary meeting was held to discuss the treatment options between the paediatrician, paediatric surgeon and interventional radiologist. The child was a poor surgical candidate as he was severely malnourished with a fairly central lesion in the right lobe of the liver. Therefore, a decision was made to attempt to treat at least the arterial inflow via an endovascular approach.

The procedure was performed under general anaesthesia. Under ultrasound guidance, a $4 \mathrm{Fr} 7 \mathrm{~cm}$ hydrophilic sheath (Angiogate TransRadial Introducer Kit by Kimal, United Kingdom) was placed retrogradely into the right common femoral artery. Intraarterial hourly heparin bolus was administered to prevent thrombosis at the access site and lower limb. Access to the celiac axis and then common hepatic artery was obtained using hydrophilic standard angled guide wire (Merit Laureate by MeritMedical, Ireland) and 4 Fr Cobra 2 catheter (Performa Angiographic catheter by MeritMedical, Mexico). The left intrahepatic arterioportal malformation was demonstrated receiving blood supply from the left hepatic artery and draining into left portal venous vein (Figure 3 ).

A decision was made to perform coil embolization of the main feeder as distally possible using a $2.4 \mathrm{Fr}$ microcatheter (Progreat by Terumo) to deploy a $4 \mathrm{~mm}$ detachable micro-coils (Concerto by Medtronic, The Netherlands). There was some flow still noted and hence a second $5 \mathrm{~mm}$ detachable micro-coil (Concerto by Medtronic, The Netherlands) was put in place which stopped the flow (Figure 4).

There was good outcome with one tiny feeder opening immediately post embolisation. digital subtraction angiography of the superior mesenteric artery showed no additional supply to the fistula.

An ultrasound scan performed 1 week after the procedure confirmed thrombosis of the fistula with antegrade flow in the portal vein (Figure 4). This was maintained on further follow up scans.

A further hospital admission 2 months after the procedure was necessary to manage refeeding syndrome. However, the patient did well clinically for a number of months once discharge, gaining significant weight and no further melaena. Unfortunately, he succumbed 8 months after the procedure secondary to an unrelated, acute respiratory illness.

\section{Discussion}

Vascular malformations are classified into fast flow, slow flow, and combined forms according to the Mullicken and Glowacki classification. Fast flow vascular malformations include the arteriovenous malformations and arterioportal fistulas. Slow flow includes portosystemic shunts, venous and lymphatic malformations. In our case, this was a fast flow arterioportal fistula. They can also be subclassified depending on symptomatology into Type 1 as small peripheral asymptomatic and like our case which is a Type 2-large central fistula causing physiological insult (6). 
Although rare, there have been cases similar to ours that have been reported. The initial modality of imaging is doppler ultrasound where there is enlargement of the hepatic artery and dilatation of the segment of the portal vein where the fistula is located (7). Pulsatile hepatofugal flow in the portal vein and color speckling in the hepatic parenchyma adjacent to the fistula may also be seen (8).

The endpoint for treatment is restoration of normal flow dynamics in the hepatic artery and portal venous system. Treatment options are often multi-staged including embolization of the feeding artery especially if a single one is identified with possible subsequent surgery involving hepatic artery ligation/resection of lesion especially if new feeders open as a result of embolization (9). For those patients who are unable to control symptoms, liver transplantation can be considered if available in the healthcare setting.

Previously, hepatic artery ligation was considered the sole intervention, but now interventional radiology has the advantage of offering minimally invasive technique which further translates to reduced mortality and morbidity with shorter in patient stay and fewer complications. The downside of the interventionalguided embolization is that it can be limited by variant anatomy, tortuosity of vessels and elongation of the vessels die to a high dynamic flow (10). The access can be transfemoral and in cases where this fails, a transhepatic approach can be attempted. The latter is often fraught with more complications including peritoneal hemorrhage, fistulization, pneumothorax (10).

The choice of embolization agent depends on the type of arterioportal malformation, size and number of the feeding arteries, location of the fistula which further determines the accessibility (10). One of the main factors especially in developing countries, is availability as in our case where we used the metallic coils which is the most commonly used agent. Other options include Amplatzer plug, $N$-butyl cyanoacrylate, polyvinyl alcohol (PVA) or microsphere (11). The Amplatzer plug is also popular as it can be used solely for occlusion of large vessels (10). PVA particles in general are not the preferred embolic agent as there is a large risk of non-target embolization into the venous system in high flow malformations. One study has reported successful embolization of larger arteriorportal malformations with detachable balloons which may be used either alone or in combination with other embolization agents (11).

One of the complications to consider include migration of the embolization agent past the site of the arterioportal malformation and into a distal tributary of the portal vein which may cause portal venous thrombosis and subsequent infarction. The risk for migration of embolization increases with increasing size of the arterioportal malformation and high flow rates (10).

The prognosis if left untreated results in arterialization of the portal vein with early onset of portal hypertension, hepatoportal sclerosis and fibrosis of the portal radicles further contributing to portal hypertension and fatal hepatic necrosis.

\section{Conclusion}

Hepatoportal fistula, a rare hepatic vascular malformation, has limited treatment options which can further be restricted by overall patient wellness. Minimally invasive endovascular treatment options can 
offer a high rate of success and reverse the morbidity associated with the disease as was seen with our case.

\section{Abbreviations}

PVA

Polyvinyl Alcohol

CT

Computer Tomography

\section{Declarations}

- Ethical approval and consent: Ethics approval and consent for publication was obtained with Ref: 2021/IERC-122 (v1). The Aga Khan University IERC committee.

- Availability of data and materials was sought from the records office- Aga Khan University Hospital.

- Competing interests: No competing interests.

- Funding: No Funding was received.

- Authors contributions: Each of the authors contributed equally to this publication. Dr. Ravjit Sagoo performed the procedure on the patient.

- Acknowledgements: None

- Author's information:

Poonamjeet Loyal: Radiology Resident, Radiology Department, Aga Khan University Hospital Nairobi, Kenya.

Ravjit Sagoo: Assistant Professor and Consultant Interventional Radiologist, Radiology Department, Aga Khan University Hospital Nairobi, Kenya.

\section{References}

1. Zhang DY, Weng SQ, Dong L et al (2015) Portal hypertension induced by congenital hepatic arterioportal fistula: Report of four clinical cases and review of the literature. World J Gastroenterol 21:2229-2235

2. Burrows PE, Dubois J, Kassarjian A (2001) Pediatric hepatic vascular anomalies. Pediatr Radiol 31:533-545

3. Davenport M, Redkar R, Howard ER et al (1999) Arterioportal hypertension: a rare complication of partial hepatectomy. Pediatr Surg Int 15:543-545

4. Tanaka H, Iwai A, Sugimoto H et al (1991) Intrahepatic Arterioportal Fistula after Blunt Hepatic Trauma. J Trauma Inj Infect Crit Care 31:143-146 
5. Vauthey JN, Tomczak RJ, Helmberger T et al (1997) The arterioportal fistula syndrome: clinicopathologic features, diagnosis, and therapy. Gastroenterology 113:1390-1401

6. Guzman EA, McCahill LE, Rogers FB (2006) Arterioportal Fistulas: Introduction of a Novel Classification With Therapeutic Implications. J Gastrointest Surg 10:543-550

7. Mora P, Rábago L, Gea F et al (1992) Sonographic features of arterioportal fistula. J Clin Ultrasound 20:291-293

8. Mallant $M$, van den Berg F, Verbeke $J$ et al (2007) Pulsatile Hepatofugal Flow in the Portal Vein: Hallmark of a Congenital Hepatoportal Arteriovenous Fistula. J Pediatr Gastroenterol Nutr 44:143145

9. Akpek S, llgıt ET, Çekirge S et al (2001) High-flow arterioportal fistula: treatment with detachable balloon occlusion. Abdom Imaging 26:277-280

10. Oguslu U, Uyanik SA, Gümüş B (2019) Endovascular treatment of hepatic arterioportal fistula complicated with giant portal vein aneurysm via percutaneous transhepatic US guided hepatic artery access: a case report and review of the literature. CVIR Endovasc 2:39

11. Tasar M, Gulec B, Bozlar U et al (2005) Intrahepatic arterioportal fistula and its treatment with detachable balloon and transcatheter embolization with coils and microspheres. Clin Imaging 29:325-330

\section{Figures}

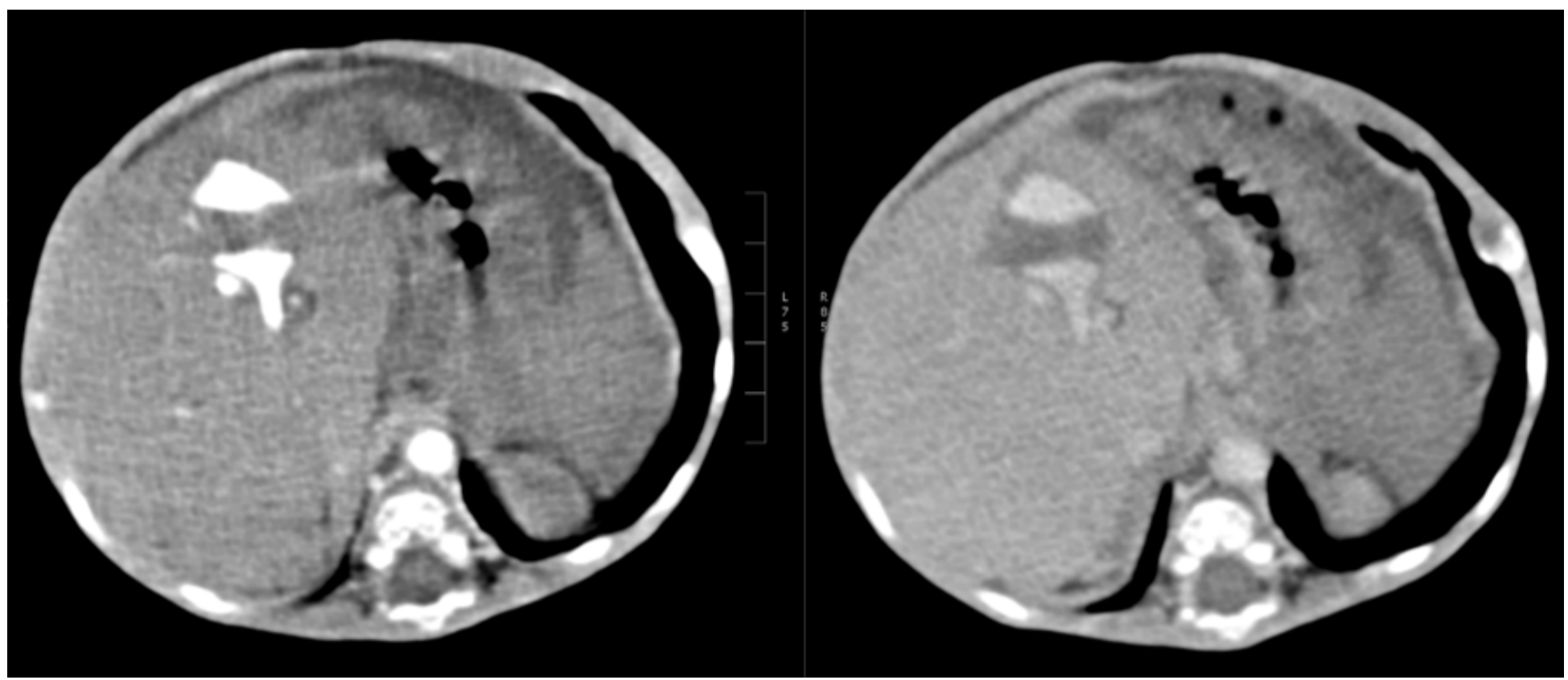

\section{Figure 1}

Axial CT arterial and portovenous images through the right lobe of the liver showing a tubular structure with early arterial filling and portovenous filling. 


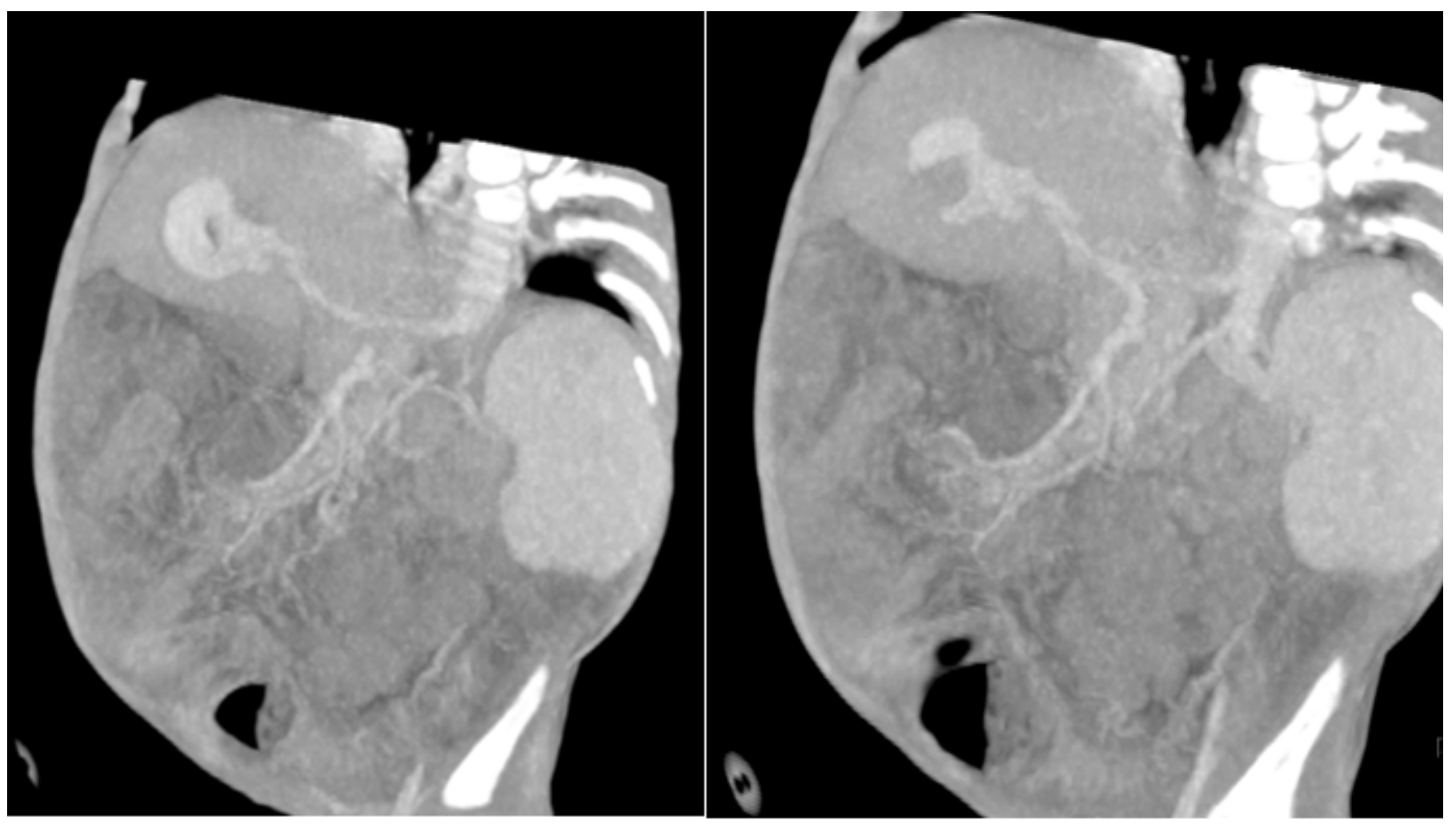

Figure 2

Coronal Maximum Intensity Projection images showing the high flow fistula with arterial supply from the left hepatic artery (left) and venous drainage into the left portal vein (right)

\section{Figure 3}

Digital subtraction angiography images demonstrating the high flow hepatic fistula with arterial supply from the left hepatic artery and venous drainage into the left portal vein

\section{Figure 4}

Endpoint achieved with metallic coil inserted showed no flow(left) and post procedure ultrasound showed intramural thrombus (right) 